\title{
L'ÉCRITURE DU FÉMININ ET LE MANQUE FONDAMENTAL
}

\author{
Leonardo Alexander do Carmo SILVA ${ }^{1}$
}

Résumé : Le Ravissement de Lol V. Stein, de Marguerite Duras, et A Paixão Segundo G.H., de Clarice Lispector, sont apparemment des romans très différents, mais ils nous permettent de réfléchir sur la question du féminin et plus précisément, sur l'écriture du féminin. Chaque récit est construit autour d'un événement majeur, un moment de basculement pour les personnages féminins. Pour Lol V. Stein, il s'agit du « rapt» de son fiancé par Anne-Marie Stretter, le soir du bal à T. Beach. Pour G.H., c'est la vision d'un cafard dans la chambre de bonne qui entraîne une véritable aventure existentialiste. Ces deux événements ont des conséquences dévastatrices pour les deux personnages. Leurs parcours se caractérisent par une perte ou une dissolution de l'identité, permettant voir que le féminin, dans ces romans, est associé à un manque fondamental.

Mots-clés : Marguerite Duras. Clarice Lispector. Féminin. Écriture du féminin.

Les romans de Clarice Lispector et de Marguerite Duras révèlent un profond intérêt pour l'univers féminin. Ils racontent généralement des histoires de femmes et les deux auteures ont créé toute une galerie de personnages féminins remarquables. Les écrivaines firent leur entrée dans le monde des lettres la même année, en 1943, avec Les Impudents et Perto do Coração Selvagem. Chacune se forgea très rapidement un style d'écriture unique, immédiatement reconnaissable. On dit souvent que leurs textes sont difficiles, novateurs, déroutants. En 1964, Marguerite Duras et Clarice Lispector ont publié deux de leurs romans les plus célèbres, Le Ravissement de Lol V. Stein et A Paixão Segundo G.H. Ces deux romans seront l'objet de notre étude.

Des questions comme «Qu'est-ce qu'une femme ?» ou «Que veut une femme ? sont fréquentes sous la plume ou dans la bouche des psychologues, des écrivains, des comiques, des hommes en général et aussi de quelques femmes. Nombreux sont les discours sur l'essence du

\footnotetext{
${ }^{1}$ Paris 3 - Université Sorbonne Nouvelle - Département Études Ibériques et Latino-Américaines. Paris - France. 75231 - leonardoalexandersilva@ gmail.com

http://dx.doi.org/10.21165/gel.v13i2.1423
}

Revista do GEL, São Paulo, v. 13, n. 2, p. 237-248, 2016 
féminin, la femme et la féminité étant souvent associées à un mystère fondamental, indéchiffrable : «N'importe quelle femme est plus mystérieuse qu'un homme. N'importe laquelle »(DURAS apud ANDERSON, 1995, p.11). Freud, dans La question de l'analyse profane, exprimait en 1926 son désarroi face à la sexualité féminine : «La vie sexuelle de la femme adulte est encore un continent noir pour la psychologie » (FREUD, 1985, p. 75). Il a emprunté cette expression à J. R. Stanley explorateur de l'Afrique, des forêts noires, impénétrables et hostiles.

Tout un discours sur la femme, ce « continent noir », a été établi par des hommes qui, historiquement, ont toujours eu accès à la parole et à l'écriture. Quelques écrivaines féministes, comme Hélène Cixous et Catherine Clément, n'ont pas cessé de dénoncer le fait qu'une «mythologie » sur la femme avait été construite à partir d'un regard masculin fondé sur une incompréhension radicale, un regard plein de clichés et de préjugés. Dans le domaine littéraire, longtemps dominé par les hommes, ce regard biaisé s'est évidemment fait remarquer.

Il est intéressant, par conséquent, d'étudier comment deux auteures contemporaines, témoins de l'évolution du statut de la femme dans la société (la révolution sexuelle, le féminisme, etc.), ont représenté l'univers féminin dans leurs ouvrages. L'étude du Ravissement de Lol V. Stein et d'A Paixão Segundo G.H. nous permettra d'analyser comment le féminin se manifeste dans les textes de Marguerite Duras et de Clarice Lispector. Lol V. Stein et G.H. occupent une place importante dans la galerie des personnages durassiens et clariciens. Selon Duras, toutes les femmes des ses livres, « quel que soit leur âge, découlent de Lol » (DURAS apud TON-THAT, 2005, p. 83). La même chose pourrait être dite à propos de G.H., considérée par quelques critiques, comme Benedito Nunes, comme un personnage central de l'œuvre de Lispector. Ces deux héroïnes sont des archétypes du féminin dans l'œuvre de Duras et de Lispector. Dans A Paixão Segundo G.H., le personnage éponyme fait quelques réflexions sur sa condition de femme :

Ter feito escultura durante um tempo indeterminado e intermitente também me dava um passado e um presente que fazia com que os outros me situassem: a mim se referem como a alguém que faz esculturas que não seriam más se tivesse havido menos amadorismo. Para uma mulher essa reputação é socialmente muito, e situou-me, tanto para os outros como para mim mesma, numa zona que socialmente fica entre mulher e homem. O que me deixava muito mais livre para ser mulher, já que eu não me ocupava formalmente em sê-lo. (APSGH, p. 25)

L'indépendance financière, le fait d'être célibataire et de ne pas avoir d'enfants donnaient à G.H. la liberté d'être une femme dans son essence, sans être définie par les rôles 
sociaux d'épouse ou de mère. Pour G.H., la féminité était un don, elle lui venait naturellement : «E quanto a homens e mulheres, que era eu? Sempre tive uma admiração extremamente afetuosa por hábitos e jeitos masculinos, e sem urgência tinha o prazer de ser feminina, ser feminina também me foi um dom » (APSGH, p. 28).

Dans le roman de Duras, lorsque le narrateur homodiégétique, Jacques Hold, essaie de se rapprocher du «mystère » qui entoure l'existence de Lol V. Stein, il franchit le « territoire du féminin », un domaine étranger, ce qui provoque l'étonnement de Tatiana : "Comment savez-vous ces choses-là sur Lol? Elle veut dire : comment les savez-vous à la place d'une femme? A la place d'une femme qui pourrait être Lol? » (LRLVS, p. 151). Jacques Hold semble devenir un initié dans cet univers féminin et il est « contaminé » par la féminité : «Tout à coup, voici leurs voix entrelacées, tendres, dans la dilution nocturne, d'une féminité pareillement rejointe en moi » (LRLVS, p. 92).

Quand G.H. parle d'elle-même, à la troisième personne, elle se désigne souvent par l'expression «cette femme »: «Levantei-me enfim da mesa do café, essa mulher » (APSGH, p. 32). Cette même expression est utilisée à plusieurs reprises dans le roman de Duras, comme substitut des noms des personnages féminins: «Pour nous, cette femme ment sur T. Beach, sur S. Tahla, sur cette soirée » (LRLVS, p. 106). Madeleine Borgomano, universitaire et écrivaine, ayant beaucoup étudié l'œuvre de Duras, affirme que l'usage que l'auteure française fait du signifiant «femme » dans ses ouvrages est très singulier (et la même chose pourrait être dite à propos de Clarice Lispector) :

Traitant le signifiant "femme" comme une sorte de nom propre - signifiant vide au départ, comme le sont les noms propres de fiction - elle laisse l'écriture en produire peu à peu les multiples signifiés : ainsi se trouvent écartés, relégués, les mythes et les essences : le féminin refusant d'être un objet définissable, devient une force en mouvement, que l'on ne peut saisir que dans sa dynamique. (BORGOMANO, 1984, p. 59)

Dans l'imaginaire collectif, la féminité est souvent associée à la maternité. La gestation est généralement considérée comme l'expérience qui définit l'être féminin : «Virtuellement ou réellement mères, toutes les femmes ont quand même une expérience de l'intérieur, une expérience de la capacité d'autre, une expérience de l'altération non négative par de l'autre, de la bonne réceptivité »(CIXOUS, 1989, p. 141). Dans Le Ravissement de Lol V. Stein et A Paixão Segundo G.H, les figures maternelles sont assez problématiques.

À travers Lol, mère indifférente, et G.H., mère « interrompue » (puisqu'elle a avorté), Duras et Lispector mettent en scène deux personnages féminins qui ne trouvent pas leur 
épanouissement dans la maternité. Mère de trois filles, Lol V. Stein semble avoir une relation assez distante avec ses enfants, une relation marquée par l'indifférence : «Elle ne bouge pas, absente, elle ne parle pas aux enfants, les enfants non plus ne lui adressent pas la parole » (LRLVS, p. 129). Dans le Ravissement de Lol V. Stein, il n'est presque jamais question des enfants, qui ne sont même pas nommés. Cela peut être révélateur du regard fantasmatique du narrateur, qui ne voit pas Lol comme une mère.

L’indifférence caractérise également la relation de Lol avec sa propre mère : « La mort de sa mère - elle avait désiré la revoir le moins possible après son mariage - la laissa sans une larme » (LRLVS, p. 32). Anne-Marie Stretter, la femme qui séduit le fiancé de Lol, est aussi une mère indifférente: «Il y avait longtemps déjà que la fille de Anne-Marie Stretter avait fui. Sa mère n'avait remarqué ni son départ ni son absence, semblait-il » (LRLVS, p. 20). La seule manifestation d'amour maternel est donnée par la mère de Lol au moment où elle cherche sa fille au bal à T. Beach. Néanmoins, elle semble ensuite vouloir se débarrasser de Lol le plus vite possible, en la mariant avec un inconnu.

Dans A Paixão Segundo G.H., l'héroöne du roman a refusé la maternité dans le passé et, au moment du récit, elle regrette l'avortement :

Pela primeira vez eu sentia com sofreguidão infernal a vontade de ter tido os filhos que eu nunca tivera: eu queria que se tivesse reproduzido, não em três ou quatro filhos, mas em vinte mil a minha orgânica infernalidade cheia de prazer. Minha sobrevivência futura em filhos é que seria a minha verdadeira atualidade, que é, não apenas eu, mas minha prazerosa espécie a nunca se interromper. Não ter tido filhos me deixava espasmódica como diante de um vício negado. (APSGH, p. 120).

Cette mère « interrompue » évoque à plusieurs reprises la Vierge Marie, archétype de la maternité, à qui elle s'adresse : «Mãe: matei uma vida, e não há braços que me recebam agora e na hora do nosso deserto, amém. Mãe, tudo agora tornou-se de ouro duro » (APSGH, p. 93). L'autre grand symbole de la maternité dans le roman est le cafard («barata » en portugais, un mot féminin) que G.H. tue dans la chambre de bonne. Le personnage perçoit l'insecte comme une figure maternelle de puissante fertilité : «Os dois olhos eram vivos como dois ovários. Ela me olhava com a fertilidade cega de seu olhar. Ela fertilizava a minha fertilidade morta » (APSGH, p. 76).

G.H. compare la masse blanche qui sort du cafard au lait maternel : «Por que teria eu nojo da massa que saía da barata? não bebera eu do branco leite que é líquida massa materna? e ao beber a coisa de que era feita a minha mãe, não havia eu chamado, sem nome, de amor? » 
(APSGH, p. 164). À la fin, les deux archétypes du féminin, la Vierge Marie et le cafard, se voient confondus dans le discours de G.H., qui parodie la prière à la Vierge en s'adressant à l'insecte : «Bendito o fruto de teu ventre » (APSGH, p. 82).

Il est également intéressant d'analyser comment le corps féminin est représenté dans les romans. Chez Lispector, il n'est presque jamais question du corps de G.H. : « À mesa do café eu me enquadrava com meu robe branco, meu rosto limpo e bem esculpido, e um corpo simples » (APSGH, p. 31). Il n'y a dans le texte aucune autre description détaillée de son physique, de son apparence. Chez Duras, les traits qui distinguent Lol sont sa blondeur, sa maigreur et ses yeux très clairs, mais le narrateur avoue : « Il ne sera jamais question de la blondeur de Lol ni de ses yeux, jamais » (LRLVS, p. 79). Il s'agit de corps effacés, absents de l'écriture.

Dans A Paixão Segundo G.H., l'héroïne s'étonne de voir son corps dessiné sur le mur de la chambre (dessin laissé par la bonne). Ce dessin, une simple silhouette, lui apparaît comme l'écriture de son identité, du vide qui constitue son existence : «E fatalmente, assim como ela era, assim deveria ter me visto? Abstraindo daquele meu corpo desenhado na parede tudo o que não era essencial, e também de mim só vendo o contorno » (APSGH, p. 40). Peu à peu, G.H. commence à se rappeler de sa bonne et l'image qui lui vient est aussi celle d'une femme maigre, d'un corps sans consistance. Une «femme invisible », découvre Lol.

Dans le roman de Lispector, le cafard est associé à la féminité et il se voit attribuer quelques caractères féminins : «No entanto ei-la, a barata neutra, sem nome de dor ou de amor. Sua única diferenciação de vida é que ela devia ser macho ou fêmea. Eu só a pensara como fêmea, pois o que é esmagado pela cintura é fêmea » (APSGH, p. 92). Métonymie de la femme, la taille nous renvoie à la fécondité et à la grossesse et son étroitesse par rapport aux hanches et à la poitrine est l'une des caractéristiques sexuelles secondaires du corps féminin, ce qui le différencie du corps masculin.

$\mathrm{Si}$, dans le roman de Lispector, la taille a une fonction métonymique, dans celui de Duras c'est la «somptueuse chevelure» de Tatiana qui fonctionne comme un symbole de la féminité et de la sensualité: «Ah! tes cheveux défaits, le soir, tout le dortoir venait voir, on t'aidait » (LRLVS, p. 79). Chez Duras, le corps féminin n'est jamais vu dans son intégralité, il est souvent morcelé dans les descriptions : «Ses seins, par rapport à sa minceur, sont lourds, ils sont assez abîmés déjà, seuls à l'être dans tout le corps de Tatiana »(LRLVS, p. 64-65).

Chez Duras et Lispector, la robe fonctionne également comme un symbole de la féminité. Dans le Ravissement de Lol V. Stein, il est souvent question des robes des femmes : la robe d'Anne Marie Stretter, la robe blanche que Lol achète pour rendre visite à Tatiana, la 
robe bleue de Tatiana, parmi d'autres. Les robes servent à distinguer les femmes : « Sa robe ne resserre pas son corps comme ses austères tailleurs d'après-midi. La robe de Lol, à l'inverse de celle de Tatiana, je crois, prend son corps de près et lui donne davantage encore cette sage raideur de pensionnaire grandie » (LRLVS, p. 147). Néanmoins, les robes peuvent au contraire produire un effet de gémellité : «Elles portent toutes deux ce soir des robes sombres qui les allongent, les font plus minces, moins différentes l'une de l'autre, peut-être, aux yeux des hommes »(LRLVS, p. 147).

Dans le roman de Lispector, G.H. utilise une robe de chambre blanche qui nous rappelle la robe blanche de Lol. Cette couleur nous renvoie à l'effacement qui caractérise la personnalité des deux héroïnes. G.H. rêve, néanmoins, d'utiliser, après la fin de son récit, une robe bleue, qui représente son retour à la vie normale : «Usarei, sim, o vestido azul novo, que me emagrece um pouco e me dá cores »(APSGH, p. 162).

Le discours psychanalytique sur la sexualité féminine se base, au moins dans les travaux de Freud et de Lacan, sur l'idée d'un manque ou d'une absence. Chez Freud, le sexe féminin n'est conçu que comme absence, coupure par rapport au sexe masculin, le phallus étant le paradigme d'un manque plus fondamental, d'un manque symbolique. Lacan dira quelques décennies plus tard que « la Femme n'existe pas » et que « la femme n'est pas toute » (LACAN, 1975). Pour lui, le sexe féminin ne se définit que par le négatif, une pensée qui s'inscrit dans la logique de la castration et de l'incomplétude. Il y a un rapprochement possible entre cette tradition de pensée et Le Ravissement de Lol V. Stein et A Paixão Segundo G.H., car dans ces romans la féminité est aussi liée à un manque ou à une absence.

L'incomplétude est un trait fondamental de l'identité de G.H. et de Lol V. Stein. G.H. commence son récit par la déclaration d'une perte : «Perdi alguma coisa que me era essencial, e que já não me é mais. Não me é necessária, assim como se eu tivesse perdido uma terceira perna que até então me impossibilitava de andar mas que fazia de mim um tripé estável » (APSGH, p. 9). Cette constatation soudaine d'un manque semble être l'un des facteurs qui motivent le récit de G.H. Le personnage ne se voit pas comme une personne entière : « Não estou à altura de imaginar uma pessoa inteira porque não sou uma pessoa inteira » (APSGH, p. 16). À propos de l'écriture de Clarice Lispector, Hélène Cixous affirme : «Ce qu'elle, femme, est en train de tracer ici, dans cet hymne à la carence, c'est l'économie du bon manque : surtout n'allons pas manquer de manque. Le manque est aussi une richesse »(CIXOUS, 1989, p. 151).

Cette économie du bon manque apparaît aussi dans Le Ravissement de Lol V. Stein dans ce qui concerne la personnalité de l'héroïne du roman. Selon Tatiana Karl, « au collège [...] il manquait déjà quelque chose à Lol pour être - elle dit : là »(LRLVS, p. 12). Comparée à une 
« dormeuse débout », à une « revenante tranquille », d'une « virtualité irréprochable », Lol est perçue comme une femme inachevée, ce qui se reflète aussi dans ses phrases qui restent parfois incomplètes : «"Peut-être qu'il ne faudrait plus que je vous voie ensemble sauf" Elle a parlé rapidement » (LRLVS, p. 175). Lol V. Stein est-elle une absence présente ? Comme souligne Madeleine Borgomano, les femmes durassiennes sont sujettes, comme Lol, à des "absences" intermittentes (BORGOMANO, 1984). L'association de Lol à la couleur blanche («Elle est blanche d'une blancheur nue », p. 112) ou même à la transparence (« la transparence de son être incendié », p. 113) suggère l'effacement continuel de sa persona.

Ce qui distingue peut-être les deux personnages féminins est la conscience de cette absence. G.H. se rend compte du vide qui constitue son être à travers l'observation de quelques photographies anciennes, ce qui déclenche chez elle une crise existentielle : « Só meus retratos é que fotografavam um abismo? Um abismo. Um abismo de nada. Só essa coisa grande e vazia: um abismo » (APSGH, p. 25). La photographie révèle la «présence d'ectoplasme » de G.H. Il s'agit du portrait «d'un creux, d'un manque, d'une absence». Dans ce processus de découverte et de reconnaissance, Lol semble s'identifier à cet abîme, à ce néant : « Aquilo que eu chamava de 'nada' era no entanto tão colado a mim que me era... eu? e portanto se tornava invisível como eu me era invisível, e tornava-se o nada » (APSGH, p. 78).

Dans le roman de Duras, le lecteur n'a accès qu'au point de vue de Jacques Hold, narrateur non-omniscient. On ne peut pas affirmer avec certitude que Lol n'a pas conscience de son «absence ». Néanmoins, le récit de Hold suggère très fortement que cette femme subit une aliénation profonde et c'est, peut-être, ce trait du caractère de Lol qui attire le narrateur, comme l'affirme le mari de Tatiana Karl : «Lot V. Stein est encore malade, vous avez vu, à table, cette absence, comme c'était impressionnant, et c'est sans doute ça qui intéresse Jacques Hold» (LRLVS, p. 156). En effet, les diagnostiques sur la «maladie » de Lol sont toujours fabriqués par les autres personnages. Tatiana, par exemple, dira pour expliquer l'incomplétude de son amie que son cœur «n'était pas là » au bon endroit et que chez cette femme «la région du sentiment n'était pas pareille » (LRLVS, p. 13).

Le manque, l'absence et l'incomplétude qui caractérisent l'existence de G.H. et de Lol trouvent d'autres échos dans les romans. Chez Duras, il y a toute une isotopie de l'effacement, de la disparition et du sommeil liée au personnage principal: «Elle se lève, éteint, disparaît » (LRLVS, p. 129) ; «Elle a un corps long et beau, très droit, raidi par l'observation d'un effacement constant» (LRLVS, p. 114) ; «Ses yeux sont veloutés comme seuls les yeux sombres le sont, or les siens sont d'eau morte et de vase mêlées, rien n'y passe en ce moment qu'une douceur ensommeillée » (LRLVS, p. 83). La «virtualité constante et silencieuse », ses 
« yeux d'eau morte», sa «voix inexpressive » sont les signes d'une existence presque impersonnelle. Selon Marini, les femmes durassiennes manifestent un « refus global $»$ « $\mathrm{Ne}$ pas se laisser atteindre, ne rien sentir, et finalement, s'absenter : telles sont les réponses féminines négatives au monde tel qu'il va. » (MARINI, 1977, p. 63).

L'incomplétude et l'inconsistance de Lol se manifestent aussi dans l'ennui profond qu'elle semble éprouver : «Elle donnait l'impression d'endurer dans un ennui tranquille une personne qu'elle se devait de paraître mais dont elle perdait la mémoire à la moindre occasion » (LRLVS, p. 12). On peut également faire mention de son indifférence et de la passivité dont elle fait preuve devant les faits de la vie : «Un jour d'octobre Lol V. Stein se trouva mariée à Jean Bedford » (LRLVS, p. 31). La voix passive révèle la soumission du personnage aux décisions et aux volontés des autres.

Dans le roman de Lispector, cette impersonnalité est signalée à travers la découverte du neutre (aussi appelé « inexpressif » et « inhumain ») par G.H. : «Para ter chegado a isso, eu abandonava a minha organização humana - para entrar nessa coisa monstruosa que é a minha neutralidade viva » (APSGH, p. 97) ; «Estou tentando te dizer de como cheguei ao neutro e ao inexpressivo de mim » (APSGH, p. 99) ; «Amor neutro. O neutro soprava. Eu estava atingindo o que havia procurado a vida toda: aquilo que é a identidade mais última e que eu havia chamado de inexpressivo » (APSGH, p. 133); «Agora aquilo que me apela e me chama é o neutro. Não tenho palavras para exprimir, e falo então em neutro » (APSGH, p. 161). G.H. s'identifie au neutre mais, en plus, il devient l'objet de sa quête. Elle veut atteindre l'inexpressif.

L'absence et le manque sont aussi exprimés à travers les noms des personnages éponymes, marqués par l'abréviation. Le nom est l'un des éléments qui désignent l'identité du sujet, il sert à individualiser et aussi à indiquer l'appartenance à un groupe familial. Dans les romans, les noms troués des personnages sont en quelque sorte leurs miroirs : «E acabei sendo o meu nome. É suficiente ver no couro de minhas valises as iniciais G.H., e eis-me » (APSGH, p. 24). Dans le roman de Duras, au contraire de ce qui arrive chez Lispector, il est permis au lecteur de connaître l'intégralité du nom de Lol, Lola Valérie Stein, mais cette femme choisit de se désigner (comme pour se rebaptiser) par son nom abrégé : «Elle prononçait son nom avec colère : Lol V. Stein - c'était ainsi qu'elle se désignait »(LRLVS, p. 23). Les noms des personnages se rapprochent ainsi d'une énigme, d'une charade. Ce n'est pas par hasard que tant de critiques ont essayé de trouver des explications, des interprétations aux noms de ces personnages. L'interprétation de l'universitaire Than-Vân Ton-That à propos du nom de Lol V. Stein me semble intéressante. Elle voit dans la symétrie du nom « Lol » autour de la voyelle 
[o], le cercle vide de l'oubli, du néant. (TON-THAT, 2005, p. 77.). Quant à G.H., plusieurs critiques ont rapproché son nom à la manière dont Lispector signait souvent ses textes : C.L.

Ces noms-charades représentent le mystère qui est au centre de l'identité de Lol V. Stein et de G.H. : «Ao olhar o retrato eu via o mistério » (APSGH, p. 23) ; « Ce fut là ma première découverte à son propos ne rien savoir de Lol était la connaître déjà. On pouvait, me parut-il, en savoir moins encore, de moins en moins sur Lol V. Stein » (LRLVS, p. 81). Ces deux personnages imposent une énigme à ceux qui essayent de les comprendre : Jacques Hold et G.H. elle-même (dans un processus d'introspection). Cela nous renvoie de nouveau au discours répandu sur la femme : un être indéchiffrable (surtout pour l'homme). Ces deux héroïnes nous renvoient ainsi à la figure du Sphinx, personnage de la mythologie grecque, qui, installé sur les remparts de Thèbes, posait une énigme qu'il avait apprise des Muses, aux voyageurs qui passaient à proximité. Ceux qui n'arrivaient pas à résoudre son énigme étaient immédiatement tués et dévorés. Le Sphinx était un monstre hybride qui avait le buste de femme et son nom en grec est féminin.

Les deux romans racontent des histoires de dépersonnalisation. Duras a exploré le thème de la perte d'identité des personnages féminins dans d'autres romans comme, Détruire dit-elle (1969) et L'Amour (1971). L'auteure a avoué être fascinée par ce sujet : « Une perte progressive d'identité est l'expérience la plus enviable qu'on puisse connaître. C'est en fait ma seule préoccupation : la possibilité d'être capable de perdre la notion de son identité. C'est pour cette raison que la question de la folie me tente tellement dans mes livres » (DURAS, 1971, p. 656). Dans Le Ravissement de Lol V. Stein, le personnage éponyme est atteint d'une profonde ignorance sur son propre être : «Elle qui ne se voit pas, on la voit ainsi, dans les autres. C'est là la toute puissance de cette matière dont elle est faite, sans port d'attache singulier » $(L R L V S$, p. 54). Dans le roman de Lispector, G.H. exprime à plusieurs reprises, au long de son discours, une perte de repères par rapport à son identité : « Naquela manhã, antes de entrar no quarto, o que era eu? Era o que os outros sempre me haviam visto ser, e assim eu me conhecia » (APSGH, p. 22), «E agora o que sou? Sou: estar de pé diante de um susto » (APSGH, p. 66).

Cette absence qui caractérise l'identité des héroïnes semble être compensée par la présence d'autres corps, de doubles, de substituts. Dans son livre L'Écriture-femme, Béatrice Didier parle de la fonction des doubles des personnages féminins :

L'héroïne a souvent une sœur, une confidente, une amie proche ou lointaine qui lui sert de miroir, certes, mais dont elle est prête aussi à devenir miroir. Tantôt l'autre femme sera très proche de l'héroïne, permettant alors l'expression d'un narcissisme fondamental, tantôt, au contraire, très différente, elle permettra, par sa différence même, d'exercer un étrange tropisme, de 
révéler à l'héroïne ce qu'elle n'est pas, ce qu'elle pourrait être, ce qu'elle aimerait être. La présence de ceux deux figures opposées permet alors à la romancière d'exprimer les aspects contradictoires d'une personnalité qui ne peut parvenir à l'unité. (DIDIER,1981, p. 27).

Dans le livre La Jeune-Née, coécrit avec Catherine Clément, l'écrivaine féministe Hélène Cixous attribue à la femme la capacité de devenir une autre, «d'être possédée ou dépossédée », ainsi qu'une réceptivité à ce qui est différent ou étranger : "Par la même ouverture, qui est son risque, elle sort d'elle-même pour aller à l'autre, voyageuse de l'inexploré, elle ne dénie pas, elle approche, non pour annuler l'écart, mais pour le voir, pour faire l'expérience de ce qu'elle n'est pas, qu'elle est, qu'elle peut être » (CIXOUS ; CLÉMENT, 1975, p. 159). Ce devenir l'autre fait partie des trajectoires de Lol V. Stein et de G.H. dans les romans.

Anne-Marie Stretter et Tatiana Karl exercent la fonction de doubles, de miroirs pour Lol V. Stein. L'héroïne du roman de Duras est une femme dont l'identité bascule et l'existence vacille. L'inconsistance de son identité lui permet de n'être personne et d'être plusieurs : «Elle se croit coulée dans une identité de nature indécise qui pourrait se nommer de noms indéfiniment différents, et dont la visibilité dépend d'elle » (LRLVS, p. 41). À la fin du roman, l'héroïne utilise le nom de Tatiana Karl en plus du sien. Cette inconsistance de l'identité de Lol est associée à ce qu'elle a vécu pendant le bal, à l'expérience d'effacement et de substitution : « À mesure que le corps de la femme apparaît à cet homme, le sien s'efface, s'efface, volupté, du monde » (LRLVS, p. 50).

Au cours de son récit, G.H. annonce à plusieurs reprises avoir perdu sa constitution humaine. Elle se voit ainsi immergée dans un processus de «dépersonnalisation » et de « déshéroïsation »: «Caminho em direção à destruição do que construí, caminho para a despersonalização »(APSGH, p. 11). La crise d'identité de G.H. lui permet de prendre conscience non seulement de sa non-identité, mais aussi des rôles qu'elle jouait et des masques qu'elle portait auparavant pour pouvoir exister socialement : «Minha pergunta, se havia, não era: "que sou”, mas “entre quais eu sou” » (APSGH, p. 27), «Eu não me impunha um papel, mas me organizara para ser compreendida por mim, não suportaria não me encontrar no catálogo » (APSGH, p. 27).

Cette prise de conscience n'empêche pas G.H., dans ce processus de questionnement et de compréhension profonde, d'établir un lien complexe d'identification avec le cafard. La vision de cette altérité radicale a l'effet d'une révélation. À mesure que le personnage continue son récit, son identité semble se mêler à celle de l'insecte : «Eu, corpo neutro de barata, eu com uma vida que finalmente não me escapa pois enfim a vejo fora de mim - eu sou a barata » 
(APSGH, p. 64). Le critique et philosophe Benedito Nunes affirme que la femme et le cafard sont liés par une existence impersonnelle et qu'ils occupent le même plan ontologique dans le récit (NUNES, 1989, p. 63). Il s'agit d'une métamorphose différente de celle narrée dans le célèbre texte de Franz Kafka, car il ne s'agit pas d'une transformation physique, mais de l'abolition de la distance entre le «moi » et l'autre.

Marguerite Duras et Clarice Lispector inscrivent le féminin dans l'ordre du néant, $d u$ vide. La femme qui a toujours été l'objet des discours les plus variés, devient chez les auteures l'image d'une absence. Mères indifférentes, interrompues ; corps absents, effacés, morcelés, dans les romans, les héroïnes se caractérisent par une incomplétude, par un manque fondamental et elles sont victimes d'une souffrance et d'une folie « sans sujet », inexpressives. Le féminin ne peut être ni saisi, ni défini. Le défi imposé à l'écriture est celui de raconter l'indicible. La « passion » des personnages féminins devient donc la passion de l'écriture, qui doit rendre compte de cette impuissance du langage de dire la femme, cette absence.

SILVA, Leonardo Alexander do Carmo. Writing womanhood and the fundamental absence. Revista do GEL, v. 13, n. 2, p. 237-248, 2016.

Abstract: Le Ravissement de Lol V. Stein, by Marguerite Duras, and A Paixão Segundo GH, by Clarice Lispector, are apparently very different novels, but they allow us to think about the representation of woman and, more precisely, about womanhood writing. Each novel is built around a major event, a moment of transformation for female characters. For Lol V. Stein, it is the "abduction" of her fiancé by Anne-Marie Stretter, during a ball at T. Beach. For G. H., it is the vision of a cockroach in the maid's room that results in a true existentialist adventure. These two events have devastating consequences for both characters. Their paths are characterized by the loss or dissolution of identity. As a result, womanhood, in the novels, is associated with a fundamental absence.

Key words: Marguerite Duras. Clarice Lispector. Womanhood. Womanhood writing.

\section{Referências}

ANDERSON, S. Le Discours féminin de Marguerite Duras : un désir pervers et ses métamorphoses. Genève : Droz, 1995.

BORGOMANO, M. Une écriture féminine ? A propos de Marguerite Duras, Littérature, $\mathrm{n}^{\circ}$ 53, p. 59-68, février, 1984.

CIXOUS, H. L'Heure de Clarice Lispector. Précédé de Vivre L'Orange. Paris : Des Femmes, 1989. 
CIXOUS, H. ; CLEMENT, C. La Jeune Née. Paris : U. G. E., 10/18, 1975. p. 159.

DIDIER, B. L'Écriture femme. Paris : PUF, 1981.

DURAS, M. Interview avec Marguerite Duras et Gabriel Cousin [Março, 1971]. The French Review, vol. XLIV, n. 4. Entrevista concedida a Bettina L. Knapp.

DURAS, M. Le Ravissement de Lol V. Stein. Paris : Gallimard, 2004[1964].

FREUD, S. La question de l'analyse profane. Paris : Gallimard, 1985[1926].

LACAN, J. Encore, Séminaire XX (1972-1973). Paris : Seuil, 1975.

LISPECTOR, C. A Paixão Segundo G.H. Rio de Janeiro: Edição do autor, 1964; Rio de Janeiro: Rocco, 1998.

MARINI, M. Territoires du féminin. Paris : Éditions de Minuit, 1977.

NUNES, B. Introdução do coordenador. In: NUNES, B. (dir.). A paixão segundo G.H.

PARIS : Association Archives de la littérature latino-americaine, des 16 Caraibes et africaine du XXe siècle; Brasília : CNPQ, 1988.

TON-THAT, T.-V. Le Ravissement de Lol V. Stein de Marguerite Duras : un roman de la folie amoureuse. Paris : Éditions du temps, 2005.

Submetido em 09/04/2016

Aceito em 26/05/2016. 